\title{
Soft X-Ray CT Imaging of a Low- Aspect-Ratio Toroidal Plasma Maintained Solely by Electron Cyclotron Heating
}

\section{AUTHOR(S):}

Tanaka, Hitoshi; Takeuchi, Tetsuro; Uchida, Masaki; Maekawa, Takashi

\section{CITATION:}

Tanaka, Hitoshi ... [et al]. Soft X-Ray CT Imaging of a Low-Aspect-Ratio Toroidal Plasma Maintained Solely by Electron Cyclotron Heating. IEEE Transactions on Plasma Science 2011, 39(11): 2480-2481

\section{ISSUE DATE:}

2011-11

URL:

http://hdl.handle.net/2433/151720

\section{RIGHT:}

c 2011 IEEE. Personal use of this material is permitted. Permission from IEEE must be obtained for all other uses, in any current or future media, including reprinting/republishing this material for advertising or promotional purposes, creating new collective works, for resale or redistribution to servers or lists, or reuse of any copyrighted component of this work in other works.; この論文は出版社版でありません。引用の際には出版社版をご確認ご利用ください。; This is not the published version. Please cite only the published version. 


\title{
Soft X-ray CT Imaging of a Low-Aspect-Ratio Toroidal Plasma Maintained Solely by Electron Cyclotron Heating
}

\author{
Hitoshi Tanaka, Tetsuro Takeuchi, Masaki Uchida, Takashi Maekawa
}

\begin{abstract}
Images of soft X-ray (SX) emission in a poloidal cross section have been reconstructed by computer tomography (CT) from 80 chord-integrated signals in total obtained in a lowaspect-ratio toroidal plasma produced and maintained solely by electron cyclotron (EC) heating on the LATE device. The SX images show the heating characteristics by electron Bernstein waves under existence of multi-harmonics of $\mathrm{EC}$ resonance in the low-aspect-ratio toroidal plasma.
\end{abstract}

Electron cyclotron (EC) heating is widely used to produce and heat plasmas for processing and for nuclear fusion. In the LATE device, the spherical tokamak equilibrium configuration is formed solely by EC heating [1], which shows possibility of starting up tokamaks without center solenoids. Observation of profiles of soft X-ray (SX) bremsstrahlung emitted by EC-heated hot electrons is one of useful diagnostics to analyze the distribution profile of such electrons. This paper presents the computer tomography (CT) images in the poloidal cross section of SX emission from the low-aspect-ratio toroidal plasma maintained solely by EC heating in the LATE device.

Four SX cameras are set on the same poloidal plane in one of the toroidal sections as illustrated in Fig. 1. Each camera is pin-hole-type and consists of a 20ch-PIN-diodearray detector, pre-amplifiers and collimation slits. The sensitive photon energy range is from 0.2 to $27 \mathrm{keV}$ [2]. The spatial resolution is about $2 \mathrm{~cm}$ on the mid-plane. The chordintegrated SX signals of 80 channels in total are digitized and stored in every $10 \mu \mathrm{s}$. For toroidal plasmas with large aspect ratio, the SX emission profile in the poloidal cross section is reconstructed using least-square-fitting method and FourierBessel expansions and is expressed in good approximation by small number of terms of expansions. On the other hand, for low-aspect-ratio toroidal plasmas, Fourier-Bessel expansions are not adequate because the SX emission profile has elongation and triangularity and requires a large number of terms of expansions. Then we use the maximum entropy method [3] to reconstruct the emission profile, where the initial emission distribution is calculated based on the power law parabolic profile bounded by four elliptic arcs [4]. This

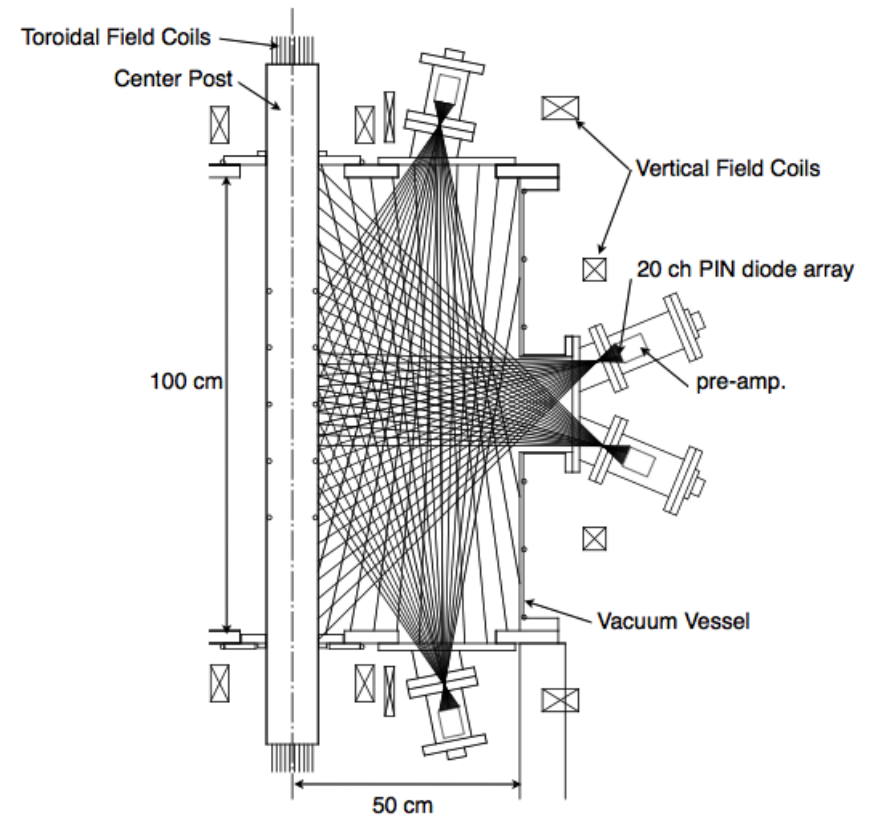

Fig. 1. Setup of four SX cameras equipped in the LATE device.

distribution model has seven fitting parameters which express position, extent, broadness and intensity. It well describes the current profile in the EC-heated plasmas in the LATE device, where the plasma current is carried by EC-heated hot tail electrons $[1,4]$. It is reasonable to use such distribution model for the SX emission profile because the bulk electron temperature is low $(<100 \mathrm{eV})$ and $\mathrm{SX}$ is mainly emitted by the tail electrons. Using the emission area bounded by the four elliptic arcs, we can diminish the spurious images on the periphery where the signal-to-noise ratio is low and the chord sections are coarse.

In the present experiments, the microwave power $(2.45 \mathrm{GHz}, 20 \mathrm{~kW}, 200 \mathrm{~ms})$ is injected in O-mode obliquely to the toroidal magnetic field by a launcher of an open circular waveguide set at a radial port. The vertical field of $\sim 20 \mathrm{G}$ is applied steadily and the toroidal field $\mathrm{Bt}$ is changed shot by shot from $192 \mathrm{G}$ to $1152 \mathrm{G}$. In a typical discharge, a toroidal plasma current begins to flow after the breakdown and increases gradually at first up to $\sim 0.8 \mathrm{kA}$, then rapidly

Manuscript received 30 November, 2010.

Authors are with Kyoto University, Graduate School of Energy Science, Kitashirakawa Oiwakecho, Kyoto, 6060-8501, Japan.

This work was supported in part by the NIFS Collaboration Research program and the Grant-in-Aid for Scientific Research of the Ministry of Education, Culture, Sports, Science and Technology of Japan.

Publisher Identifier S XXXX-XXXXXXX-X 
(a) $B_{t}=1152 G, R_{E C R}=32.9 \mathrm{~cm}$

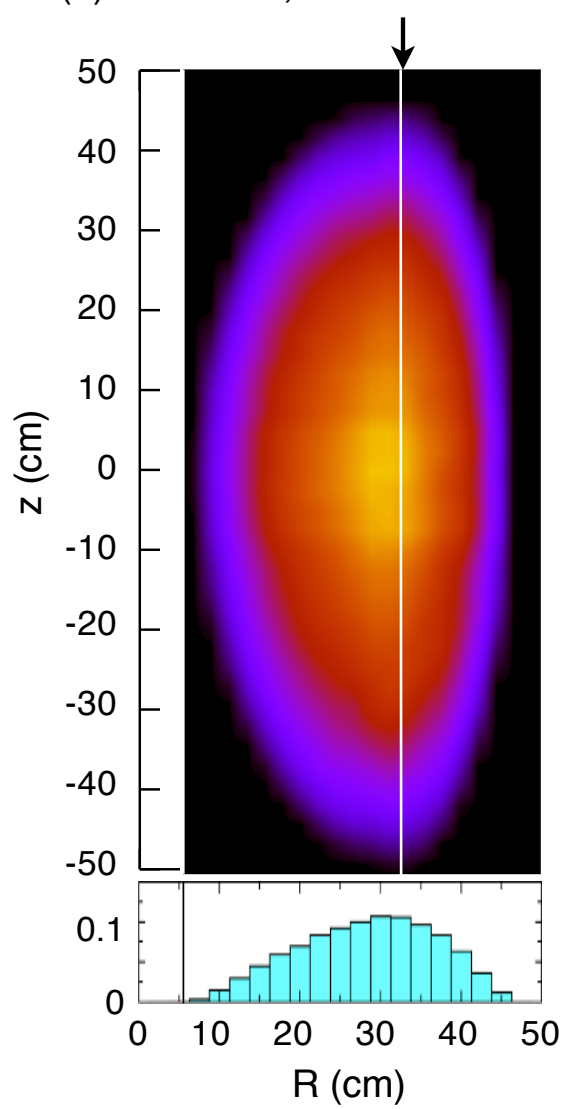

(b) $B_{t}=912 G, R_{E C R}=26.0 \mathrm{~cm}$

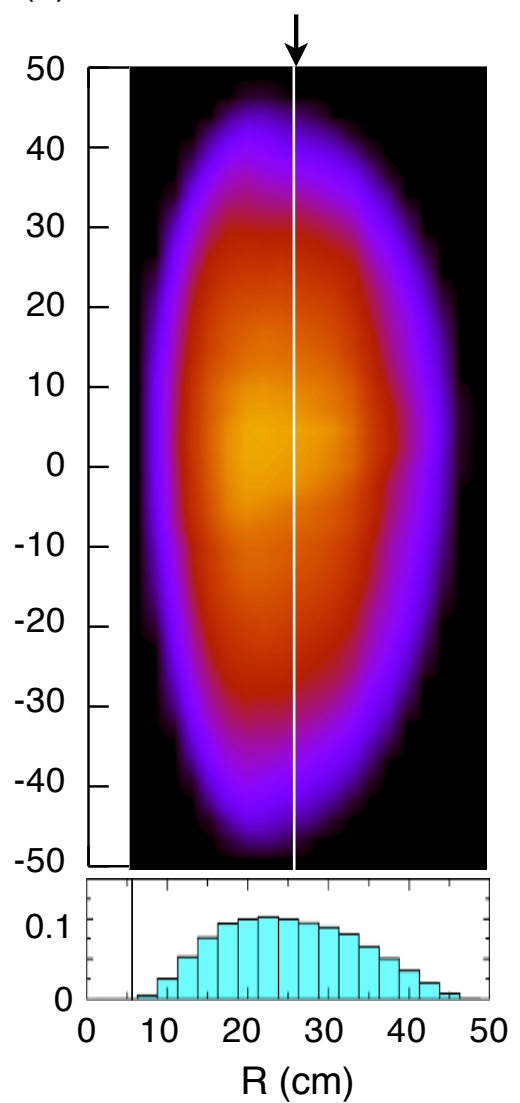

(c) $B_{t}=480 G, R_{E C R}=13.7 \mathrm{~cm}$

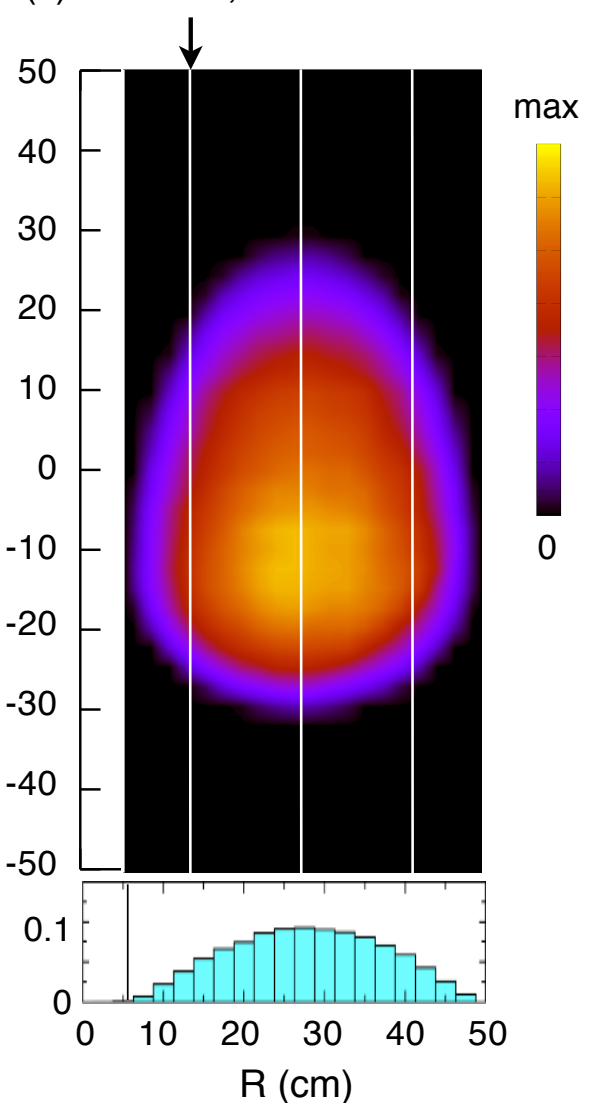

Fig. 2. Reconstructed profiles of SX emission for three different toroidal magnetic field strengths. The locations of the fundamental EC resonance layer are shown by arrows. The positions of harmonics layers of EC resonance are indicated by white lines in the images. Lower figures show the radial profile of SX relative intensity integrated along the vertical line calculated from the reconstructed emissivity and normalized by the total intensity.

increases to $\sim 2 \mathrm{kA}$ in about $3 \mathrm{~ms}$, resulting in the formation of closed flux surface [1]. The line-averaged electron density measured by the $70 \mathrm{GHz}$ microwave interferometer is $\sim 1.5 \mathrm{x}$ $10^{11} \mathrm{~cm}^{-3}$ and nearly double the plasma cutoff density, suggesting EC heating by mode-converted electron Bernstein (EB) waves. The bulk electron temperature is estimated to be $\sim 70 \mathrm{eV}$ from line-spectral intensities of different excitation states of oxygen and carbon ions. The bulk electrons' part of the poloidal beta is $\sim 0.07$, while the poloidal beta obtained with the magnetic measurement is $\sim 1.4$. The main contribution to the plasma pressure is due to high energy tail electrons heated by the EB wave. At this stage, SX emission profiles are reconstructed as shown in Fig. 2 in three different cases of $\mathrm{Bt}=$ (a) $1152 \mathrm{G}$, (b) $912 \mathrm{G}$ and (c) $480 \mathrm{G}$. In Figs. 2 (a) and (b), only the fundamental EC resonance layer exists in the vacuum vessel at the radius $\mathrm{R}_{\mathrm{ECR}}=32.9 \mathrm{~cm}$ and $26.0 \mathrm{~cm}$, respectively. The peak position of SX emission profile is around the resonance and shifts radially in accordance with the position of the resonance. In Fig. 2(c), three harmonics of EC resonance from the fundamental to the third are in the vacuum vessel and the SX emission is strong around the second EC resonance layer which locates near the center of the vacuum vessel. The SX emission profiles reflect the heating characteristics of EB waves in the low-aspect-ratio toroidal plasma where multi-layers of harmonics of EC resonance exist in the vacuum vessel.

\section{REFERENCES}

[1] M. Uchida, T. Yoshinaga, H. Tanaka and T. Maekawa, "Rapid Current Ramp-Up by Cyclotron-Driving Electrons beyond Runaway Velocity”, Phys. Rev. Letters 104, 065001 (2010).

[2] S. Yoshimura, M. Watanabe, K. Tanabe, A. Nakayama, et al., "Suppression of a Pressure Driven $\mathrm{m}=1$ mode in a Lower Hybrid Current Drive Plasma by Electron Cyclotron Heating in the WT-3 Tokamak", Phys. Plasmas 7, 276-282 (2000)

[3] K. Ertl, W. von der Linden, V. Dose and A. Weller, "Maximum Entropy Based Reconstruction of Soft X Ray Emissivity Profiles in W7-AS", Nucl. Fusion 36, 1477-1488 (1996)

[4] T. Yoshinaga, M. Uchida, H. Tanaka and T. Maekawa, "A Current Profile Model for Magnetic Analysis of the Start-up Phase of Toroidal Plasmas Driven by Electron Cyclotron Heating and Current Drive", Nucl. Fusion 47, 210-216 (2007) 